\title{
Utility of diffusion-weighted and contrast- enhanced magnetic resonance imaging in diagnosing and differentiating between high- and low-grade uterine endometrial stromal sarcoma
}

Yen-Ling Huang ${ }^{1,2}$, Shir-Hwa Ueng ${ }^{3}$, Kueian Chen ${ }^{1,2,6}$, Yu-Ting Huang ${ }^{1,5}$, Hsin-Ying Lu ${ }^{1,2,6}$, Koon-Kwan Ng ${ }^{1,5}$, Ting-Chang Chang ${ }^{4,7}$, Chyong-Huey Lai ${ }^{4,7}$ and Gigin Lin ${ }^{1,2,6^{*}}$ (D)

\begin{abstract}
Background: Endometrial stromal sarcoma (ESS) is a rare uterine malignancy that features different prognoses for its high- and low-grade subtypes. We investigated the diagnostic accuracy of magnetic resonance (MR) imaging in diagnosing and differentiating between high- and low-grade ESS.

Methods: We retrospectively reviewed the preoperative pelvic MR images of consecutive patients who received histologically confirmed diagnoses of high-grade ESS $(n=11)$ and low-grade ESS $(n=9)$ and T2-hyperintense leiomyoma $(n=16)$. Two radiologists independently evaluated imaging features in T1-, T2-, and diffusion-weighted and contrast-enhanced MR images. Statistical analysis included Mann-Whitney tests and Fisher's exact test, with sensitivity, specificity and diagnostic accuracy of imaging features.

Results: High-grade ESS was associated with significantly more extensive necrosis and hemorrhage and distinct feather-like enhancement compared with low-grade ESS ( $P<.05$ for all). The feather-like enhancement pattern yielded a diagnostic accuracy of 95\%, sensitivity of 91\%, and specificity of 100\% in differentiating high-grade from low-grade ESS. This imaging characteristic was significantly superior to the necrosis $(80 \%, P=.033)$ or hemorrhage (75\%, $P=.007)$. Both high- and low-grade ESS demonstrated T2 hypointense bands, marginal nodules, intratumoral nodules, and worm-like intra-myometrial nodules, and their tumor apparent diffusion coefficient (ADC) values were significantly lower than those of T2-hyperintense leiomyomas $(P<.001)$.
\end{abstract}

Conclusions: Diffusion-weighted MR imaging is useful in diagnosing ESS against T2-hyperintense leiomyomas, and contrast enhancement aids in further differentiating between high- and low-grade ESS.

Keywords: Endometrial stromal sarcoma, Uterine leiomyoma, Magnetic resonance imaging, Diffusion-weighted imaging, Accuracy, Grading

\footnotetext{
* Correspondence: giginlin@cgmh.org.tw

'Department of Medical Imaging and Intervention, Chang Gung Memorial

Hospital at Linkou, 5 Fuhsing St., Guishan, Taoyuan, Taiwan33382

${ }^{2}$ Imaging Core Laboratory, Institute for Radiological Research, Chang Gung

Memorial Hospital at Linkou and Chang Gung University, 5 Fuhsing St.,

Guishan, Taoyuan, Taiwan33382

Full list of author information is available at the end of the article
}

(C) The Author(s). 2019 Open Access This article is distributed under the terms of the Creative Commons Attribution 4.0 International License (http://creativecommons.org/licenses/by/4.0/), which permits unrestricted use, distribution, and reproduction in any medium, provided you give appropriate credit to the original author(s) and the source, provide a link to the Creative Commons license, and indicate if changes were made. The Creative Commons Public Domain Dedication waiver (http://creativecommons.org/publicdomain/zero/1.0/) applies to the data made available in this article, unless otherwise stated. 


\section{Background}

Uterine sarcomas are rare but aggressive, and they contribute to $3-7 \%$ of all uterine malignancy cases and to $26 \%$ of total cancer related mortality [1-3]. Endometrial stromal sarcoma (ESS) accounts for 10$21 \%$ of all uterine sarcoma cases, and the clinical presentation of ESS mimics that of benign uterine leiomyoma [1, 2]. Preoperative diagnosis of uterine ESS is critical given the emergence of nonsurgical options for benign leiomyomas, such as uterine artery embolization and high-intensity focused ultrasonography [4]. ESS is classified into high-grade and low-grade subtypes based on morphology, nuclear pleomorphism, and necrosis [5]; the subtypes have different therapeutic and prognostic profiles. Highgrade and undifferentiated endometrial sarcoma, referred to as high-grade ESS herein, behaves aggressively and is associated with a 5-year survival rate of only 33\% [6,7], whereas low-grade ESS behaves indolently and is associated with a 5-year survival rate of $91 \%$ [7]. Surgery is the primary treatment for both high-grade and low-grade ESS confined to the uterus [8]. For high-grade ESS, adjuvant systemic chemotherapy or radiotherapy is recommended, whereas adjuvant hormone therapy is recommended for lowgrade ESS [1]. Pretreatment diagnosis of ESS and differentiation between high-grade and low-grade ESS would assist clinicians in precisely tailoring the treatment plan for patients.

Magnetic resonance (MR) imaging has proven useful in the pretreatment survey of uterine malignancies [9]. With the routine and emerging advanced imaging sequences such as diffusion-weighted (DW), whose measurement were reflected by the biophysical characteristics of the tissue, rendered the discrimination of malignant from benign $[10,11]$. More than $60 \%$ of benign leiomyomas demonstrate T2 hypointensity as compared with the myometrium [12]. However, varying extent of degeneration and edema or increased cellularity of benign leiomyomas cause T2 hyperintensity, which hinders their distinction from uterine sarcomas [12]. Adding to the diagnostic difficulty is the lack of studies on differentiation between high- and low-grade ESS based on imaging [13-16]. ESS has been reported to manifest as large masses with or without evidence of myometrial invasion on MR images [13], demonstrating T2 hypointense bands [14], marginal nodules and worm-like intra-myometrial nodules [15], or projections in the vessels or along the ligaments [16]. One report showed that high-grade ESS infiltrates the myometrium more destructively than low-grade ESS [5], and another recent report suggested overlapping morphological appearance on MR images [17]. The diagnostic performance of robust MR sequences, namely contrast-enhanced (CE) MR imaging and DW MR imaging with an apparent diffusion coefficient (ADC) value, in differentiation between high- and low-grade ESS remains to be established.

We aim to investigate the diagnostic accuracy of MR imaging in diagnosing and differentiating between highgrade and low-grade ESS.

\section{Methods}

\section{Study population}

Our institutional review board approved this Health Insurance Portability and Accountability Act-compliant retrospective study, and the requirement of informed consent was waived. From a review of our pathology database records between January 2000 and December 2016, we identified 91 cases of pathologically confirmed endometrial stromal and related tumors. Among these cases, MR imaging before hysterectomy or definitive surgery was performed in 34 cases. We excluded 10 cases in which only myomectomy or hysteroscopic removal of the primary tumor for diagnostic purposes was performed without gross residual tumors on MR images. For 4 cases, MR imaging was performed before the introduction of the Picture Archiving and Communication System (PACS); therefore, the images could not be acquired. Finally, 20 cases for which preoperative MR examination was performed and images could be acquired from our current PACS were included in the final analysis. We added the MR images of benign T2hyperintense leiomyomas excluding hemorrhagic infarctions from a continuous cohort that included 126 benign leiomyomas diagnosed during 2015-2017 in patients who received complete preoperative imaging workup and surgical pathology. Figure 1 shows the flowchart of the study design.

\section{MR protocol}

Given that the study was conducted over 17 years, the MR imaging equipment and scan parameters varied. Of the 20 patients, 10 were examined using a $3 \mathrm{~T}$ MR system (6 using Skyra, Trio Tim, Siemens Medical System, Erlangen, Germany; 3 using Trio Tim, Siemens Medical System, Erlangen, Germany; and 1 using Discovery MR750, GE Medical System, Milwaukee, USA) and the rest using a $1.5 \mathrm{~T}$ MR system (Magnetom Vision, Siemens Medical System, Erlangen, Germany; Gyroscan Intera, Philips Medical Systems, Best, the Netherlands; Optima MR450w, GE Medical System, Milwaukee, USA; Signa HDxt, GE Medical System, Milwaukee, USA). In general, in the MR scans, phased-array body coils were applied to cover the entire pelvis. The imaging studies were performed during normal respiration and without the administration of premedication or antiperistaltic agents. All studies included T1- and T2-weighted spinecho sequences and CE MR images (slice thickness, 4 


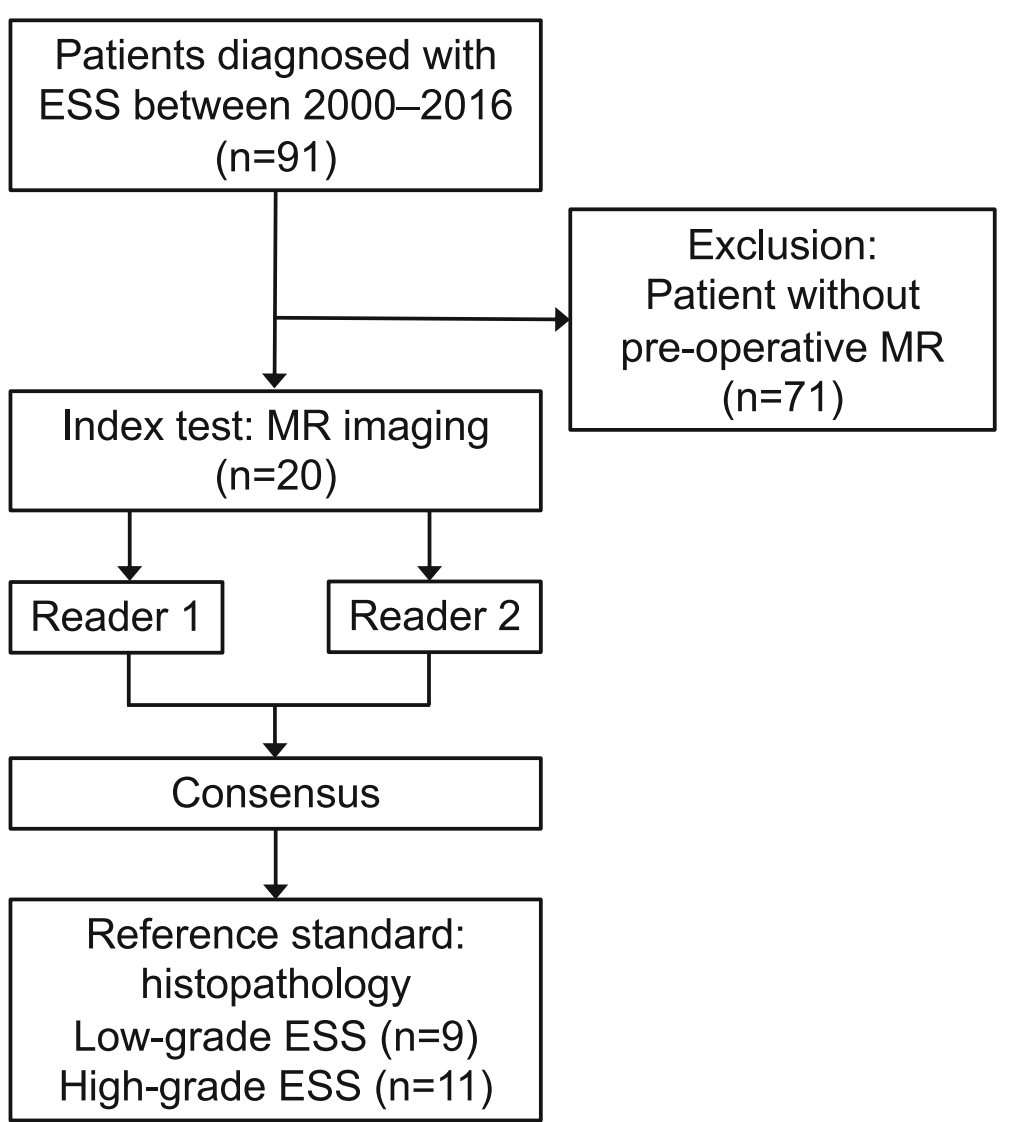

Fig. 1 Flow diagram of the study design

mm; gap, $1 \mathrm{~mm}$; matrix, $256 \times 320$; field of view, $20 \mathrm{~cm}$ ). CE MR images was acquired in the sagittal and axial planes at approximately $120-180 \mathrm{~s}$ in the equilibrium phase after an intravenous injection with the contrast medium at $0.1 \mathrm{mmol} / \mathrm{kg}$ body weight (gadopentetate dimeglumine, Magnevist, Schering, Berlin, Germany), followed by $20-\mathrm{mL}$ saline flush at a rate of $2-3 \mathrm{~mL} / \mathrm{s}$. After 2006, DW MR imaging was routinely performed using a single-shot echo-planar technique with fat suppression. ADC maps were generated from isotropic DW images with b-values of 0 and $1000 \mathrm{~s} / \mathrm{mm}^{2}$ by calculating the slope of the logarithmic decay curve for signal intensity against b-values.

\section{Imaging analysis}

Two radiologists with 12 and 3 years of experience in gynecologic radiology retrospectively and independently interpreted the MR images. All images were stored in the PACS of our hospital and were acquired for analytical purposes. Image review was performed independently using dedicated image processing software (OsiriX MD v8.0.2, Geneva, Switzerland) on an offline macOS system (MacBook Pro, Apple, Cupertino, CA), with the reviewer blinded to clinical information. Two readers independently assessed imaging parameters including tumor size, location, margin, T1 weighting, T2 weighting, $\mathrm{CE}$, and DW, and consensuses on imaging features was achieved through joint review. The worm-like nodular extension was defined as detached or discrete nodules from the primary tumor representing myometrial and lymphovascular invasion [15]. Marginal nodules were defined as nodular lesions at the tumor margin, also representing myometrial invasions of tumors [15]. Intratumoral T2 low-signal-intensity bands were scattered, preserved bundle of the myometrium [14] (Fig. 2). High signal intensities on pre-enhanced T1-weighted images were considered indicators of hemorrhage. We defined necrosis as areas of high signal intensity on T2-weighted images and the lack of enhancement after administration of the contrast medium. The feather-like enhancement was used to describe fine, wispy enhancement interspersed within tumors (Fig. 3). The ADC values of each primary tumor were measured using manually drawn regions of interest on the console with main tumors on the largest tumor plane, excluding the necrotic and nonenhanced portions. The average of the ADC values measured by the two readers independently was considered the representative $\mathrm{ADC}$ value for each tumor. Additional file 1: Table S1 details the MR imaging features. 

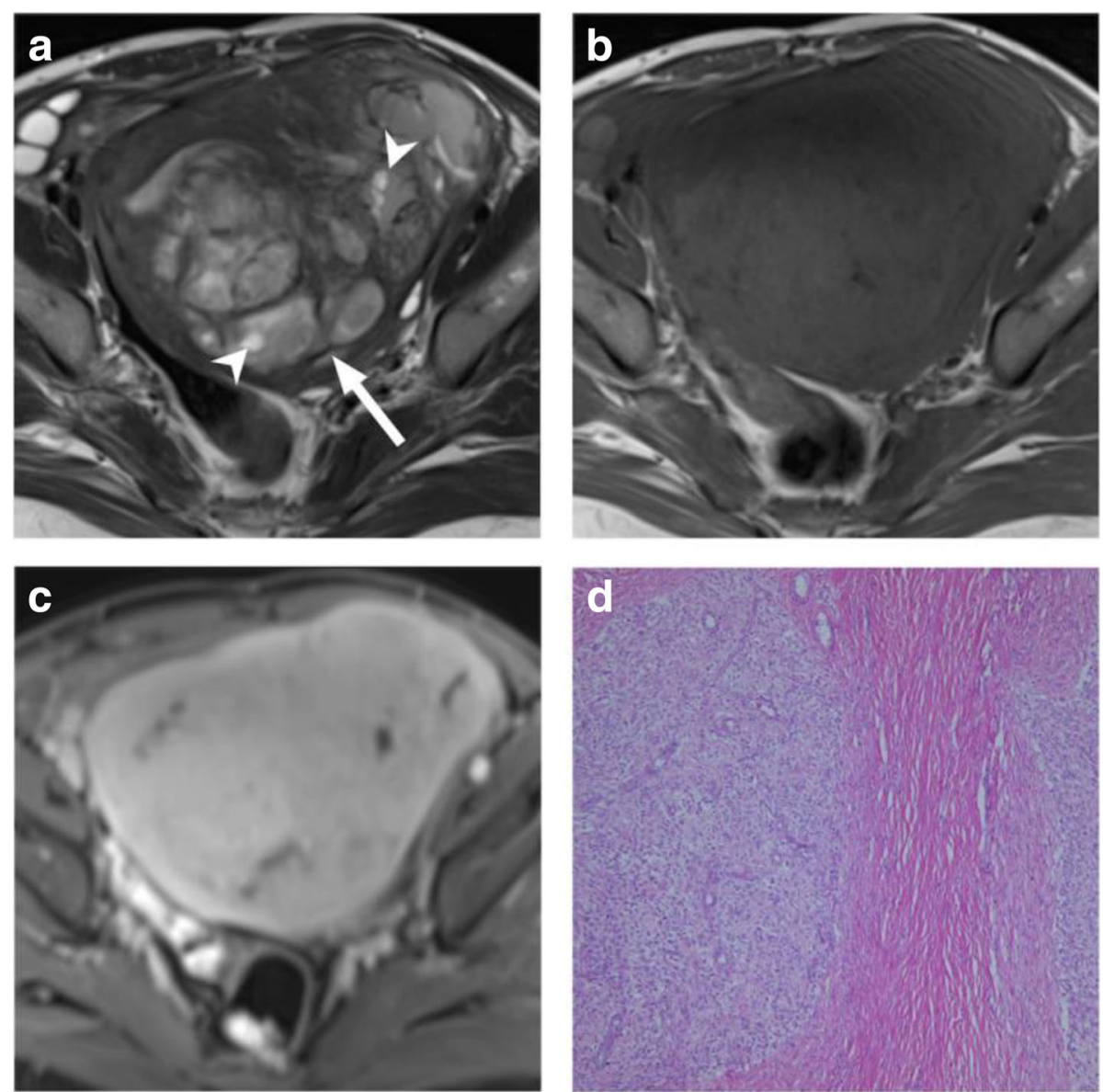

Fig. 2 A 35-year-old female patient had a 12.3-cm infiltrative tumor involving both endometrial cavity and myometrium. a Axial T2WI image shows tumor signal higher than the adjacent myometrium (arrowhead), with T2 hypointense bands interspersed within the tumor (arrow). b This tumor exhibits isointense T1 signal. c Post-contrast fat-saturated T1WI shows slightly heterogeneous enhancement. $\mathbf{d}$ H\&E stain shows irregular tumor nests of blue cells permeating the myometrium without an associated stromal reaction. Pathology report yielded a low-grade endometrial stromal sarcoma

\section{Histopathologic analysis}

The reference standard used in this study was surgical histopathology, which was reviewed by a pathologist with 13 years of experience in gynecologic pathology. The pathological findings including tumor size, differentiation, nuclear atypia, mitotic count, round cell morphology, and necrosis were reviewed and evaluated on hematoxylin-eosin-stained slides. Histopathologic diagnosis was performed based on the 2014 World Health Organization (WHO) classification criteria [18]. The clinical information and MR results were made available to the assessors of the reference standard.

\section{Statistical analysis}

We analyzed the data using the Statistical Package for Social Sciences version 11 (SPSS, Chicago, IL). The Mann-Whitney $U$ test and Fisher's exact test were used to compare the pathological findings and
MR imaging features between all ESS and T2-hyperintense leiomyomas and between high-grade and low-grade ESS. A $P$ value less than .05 was considered statistically significant. Reader agreement on imaging features and interobserver variability were analyzed using weighted kappa statistics $(0.00 \leq \kappa<0.40$ indicated poor agreement; $0.40 \leq \kappa \leq 0.70$ indicated moderate agreement; $0.70 \leq \kappa \leq 0.90$ indicated good agreement; and $\kappa>0.90$ indicated excellent agreement). The overall sensitivity, specificity, and diagnostic accuracy of each imaging characteristic were determined based on the histopathological reference, and the data are represented in $95 \%$ confidence intervals. The McNemar test was used to compare the sensitivity, specificity, and diagnostic accuracy of imaging features that showed a significant difference between high-grade and low-grade ESS. Areas under the receiver operating characteristic curve (AUCs) were calculated to compare diagnostic performance in each group. 

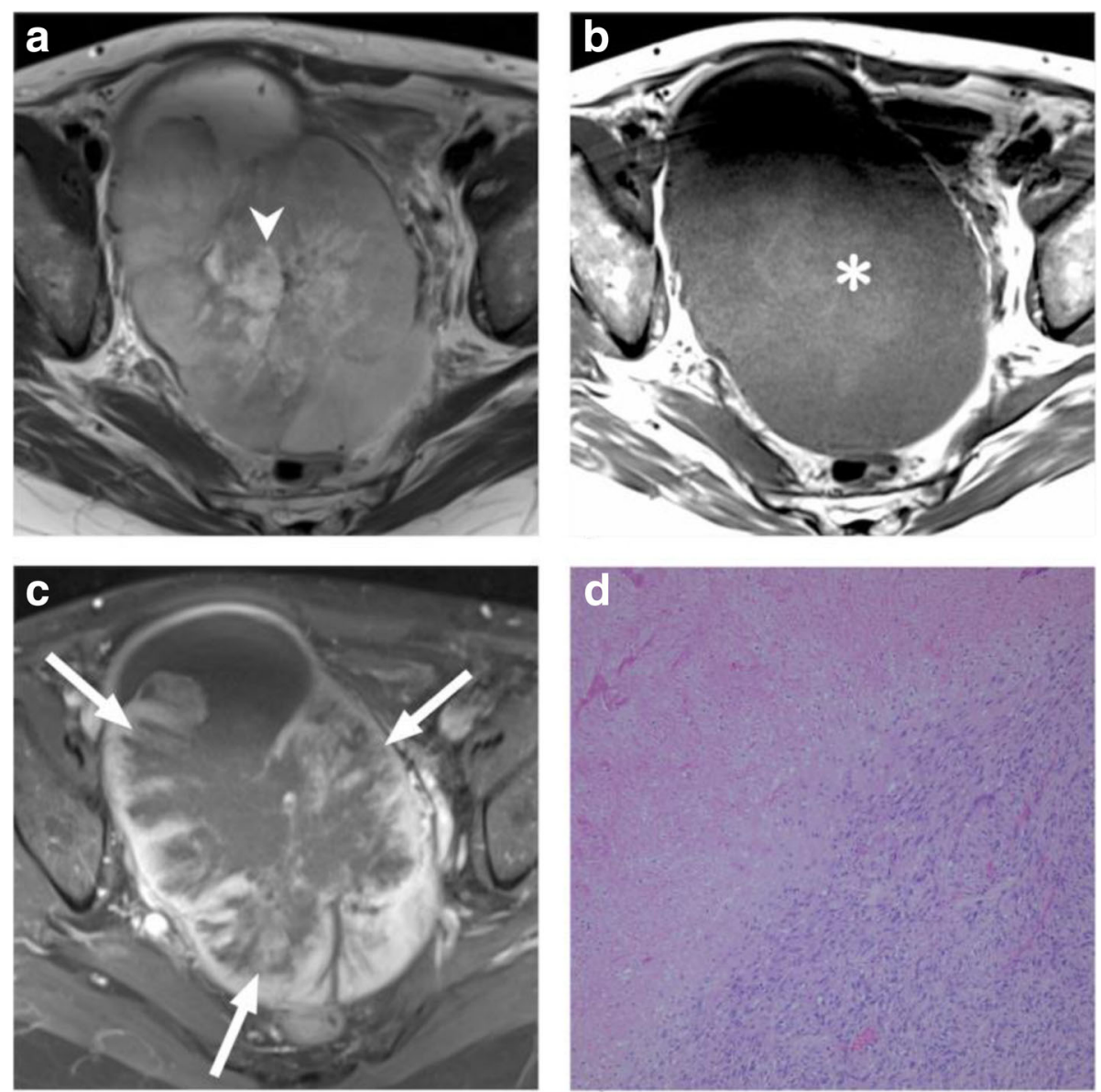

Fig. 3 A 61-year-old woman had a 14.5-cm infiltrative tumor involving both endometrial cavity and myometrium. a Axial T2WI image shows hyperintensity within the solid tumor representing necrosis (arrowhead). b Axial T1WI image demonstrates hyperintensity representing hemorrhage (asterisk). c Post-contrast fat-saturated T1WI shows feather-like enhancement (arrow) within the tumor. $\mathbf{d}$ H\&E stain shows small round cells with mitotic figures, as well as extensive necrosis and intratumoral hemorrhage. Pathology report yielded a high-grade endometrial stromal sarcoma

\section{Results}

\section{Patients}

Eleven patients with high-grade ESS and 9 with lowgrade ESS were identified. Table 1 summarizes patient demographics. Patients with high-grade ESS were significantly older than patients with low-grade ESS (median age, 64 vs. 42 years, $P<.001$ ); however, the ages overlapped substantially. The interval between the MR examination and surgery ranged from 0 to 30 days (median, 11 days). No remarkable adverse events were recorded during MR examination.

\section{MR imaging characteristics of T2-hyperintense leiomyomas and ESS}

The tumor morphology of pathologically confirmed benign T2-hyperintense leiomyomas differed significantly from that of ESS on MR imaging, as summarized in Table 2. None of the benign leiomyomas demonstrated infiltrative tumor margins, worm-like intramyometrial nodules, marginal nodules, or feather-like enhancement. In contrast to ESS, only a small percentage of leiomyomas showed intralesional necrosis and hemorrhage $(P=.001$ and .002 , respectively). Benign leiomyomas showed enhancement that was the same as that in the normal myometrium (Fig. 4), whereas ESS showed less enhancement than that in the adjacent unaffected myometrium $(P=.001)$. The ADC value was significantly lower in ESS than in T2-hyperintense leiomyomas $\left(P<.001 ;\right.$ mean \pm standard deviation, $0.99 \pm 0.13 \times 10^{-3}$ $\left.\mathrm{mm}^{2} / \mathrm{s}\right)$ and low-grade ESS $\left(1.09 \pm 0.120 \times 10^{-3} \mathrm{~mm}^{2} / \mathrm{s}\right.$, $P<.001)$.

\section{MR imaging characteristics of high-grade and low-grade ESS}

Table 3 summarizes the MR imaging characteristics for our 20 cases. Reader agreement was good to excellent for tumor size, location, involvement, and MR imaging features. Both high-grade and low-grade ESS appeared 
Table 1 Demographics of the study participants

\begin{tabular}{|c|c|c|c|}
\hline & T2-hyperintense leiomyoma & High-grade ESS & Low-grade ESS \\
\hline$n$ & 16 & 11 & 9 \\
\hline \multicolumn{4}{|l|}{ Age (year) } \\
\hline Median (range) & $43(30-49)$ & $64(45-78)$ & $42(29-55)$ \\
\hline \multicolumn{4}{|l|}{ Menopausal status } \\
\hline Premenopausal & $16(100.0)$ & $1(9.1)$ & $8(88.9)$ \\
\hline Postmenopausal & $0(0.0)$ & $10(90.9)$ & $1(11.1)$ \\
\hline \multicolumn{4}{|l|}{ FIGO staging } \\
\hline । & N/A & $5(45.5)$ & $8(88.9)$ \\
\hline$\|$ & $\mathrm{N} / \mathrm{A}$ & $0(0.0)$ & $0(0.0)$ \\
\hline III & $\mathrm{N} / \mathrm{A}$ & $4(36.4)$ & $1(11.1)$ \\
\hline IV & N/A & $2(18.2)$ & $0(0.0)$ \\
\hline \multicolumn{4}{|l|}{ Treatment } \\
\hline Surgery alone & $16(100.0)$ & $3(27.3)$ & $5(55.6)$ \\
\hline Surgery with adjuvant treatment & $\mathrm{N} / \mathrm{A}$ & $7(63.6)$ & $4(44.4)$ \\
\hline CCRT alone & $\mathrm{N} / \mathrm{A}$ & $1(9.1)$ & $0(0.0)$ \\
\hline
\end{tabular}

Data in parentheses are percentages

CCRT Combined chemoradiotherapy

as largely solid and occasionally cystic tumors located at the uterine fundus or the body, and they were either confined within the myometrium or involved both the endometrium and myometrium and had demarcated or infiltrative margins. The sizes of high-grade ESS tumors were larger than those of low-grade ESS tumors (11.25 $\mathrm{cm}^{3}$ vs. $\left.7.76 \mathrm{~cm}^{3}\right)$; however, the difference was not statistically significant $(P=.056)$. High-grade and low-grade ESS did not show significant differences in the presence of worm-like, marginal, or intramural nodules. T2 hypointense bands appeared in all high-grade and lowgrade ESS tumors. The three imaging features that

Table 2 MR imaging characteristics between endometrial stromal sarcomas (ESS) and T2-hyperintense leiomyomas

\begin{tabular}{|c|c|c|c|}
\hline Parameters & ESS & T2-hyperintense leiomyoma & $P$ value \\
\hline$n$ & 20 & 16 & \\
\hline Tumor size $(\mathrm{cm})$ & $9.68 \pm 3.59$ & $11.66 \pm 4.45$ & 0.265 \\
\hline ADC value $\left(\times 10^{-3} \mathrm{~mm}^{2} / \mathrm{sec}\right)$ & $1025.61 \pm 130.31$ & $1348.19 \pm 162.15$ & $<0.001 *$ \\
\hline Margin & & & $0.014^{*}$ \\
\hline Well-defined & $12(60.0)$ & $16(100.0)$ & \\
\hline III-defined & $8(40.0)$ & $0(0.0)$ & \\
\hline Worm-like nodules & $8(40.0)$ & $0(0.0)$ & $0.014 *$ \\
\hline Marginal nodules & $14(70.0)$ & $0(0.0)$ & $<0.001 *$ \\
\hline Intratumoral nodules & $16(80.0)$ & $7(43.8)$ & 0.057 \\
\hline T2 hypointense bands & $20(100.0)$ & $1(6.3)$ & $<0.001 *$ \\
\hline Feather-like enhancement & $10(50.0)$ & $0(0.0)$ & $0.003 *$ \\
\hline Necrosis & $13(65.0)$ & $1(6.3)$ & $0.001 *$ \\
\hline Hemorrhage & $14(70.0)$ & $2(12.5)$ & $0.002 *$ \\
\hline Tumor enhancement & & & $0.001 *$ \\
\hline Less than myometrium & $15(75.0)$ & $5(31.3)$ & \\
\hline Equal to myometrium & $1(5.0)$ & $10(62.5)$ & \\
\hline Greater than myometrium & $4(20.0)$ & $1(6.3)$ & \\
\hline
\end{tabular}



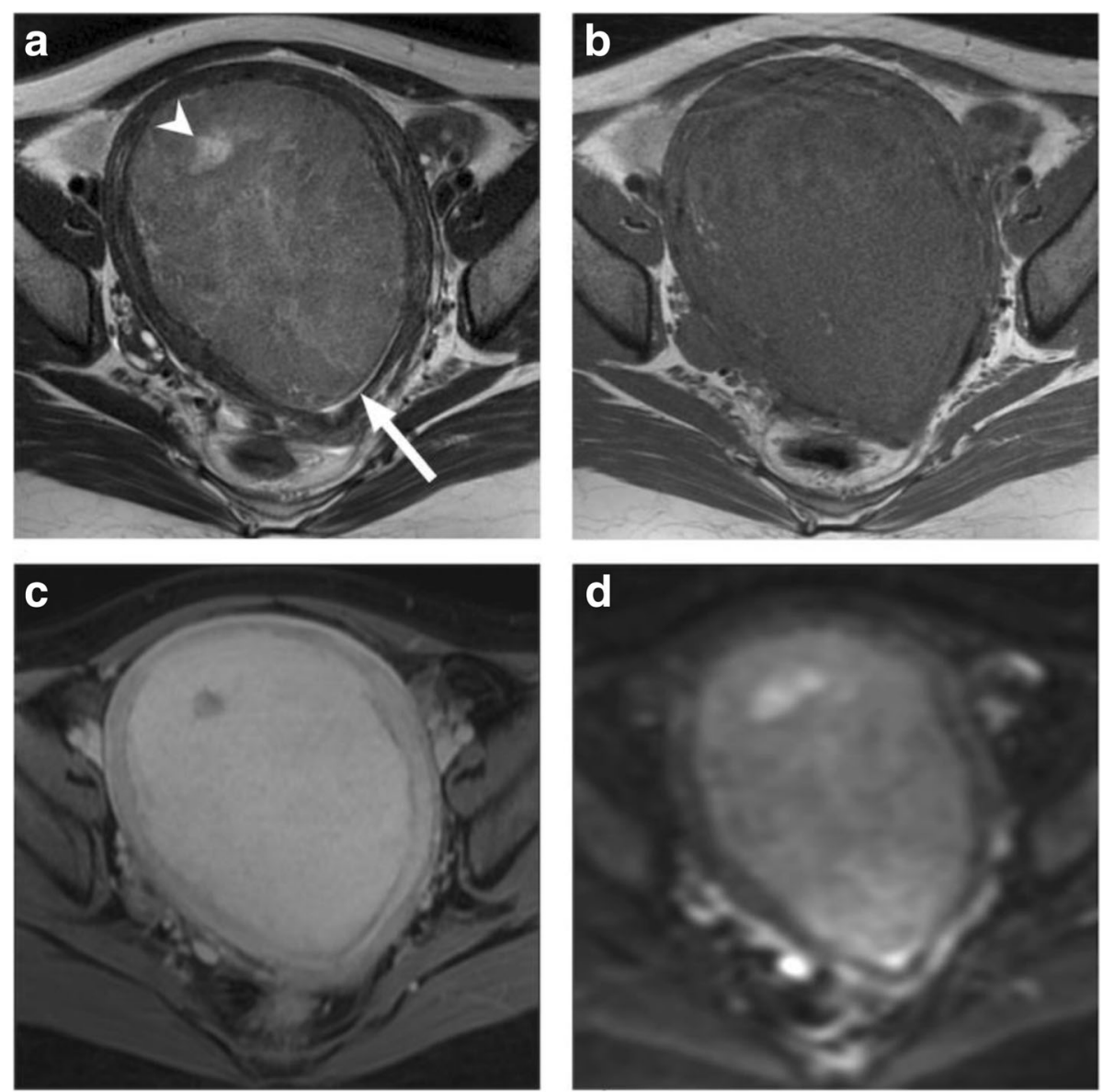

Fig. 4 A 48-year-old female had a 12.6-cm intrauterine leiomyoma, arising from the anterior wall of the uterus, compressing the endometrial cavity (arrow). The tumor exhibits higher signal intensity than that of the myometrium on axial T2WI image (a), with focus of edema or necrosis (arrowhead), isointensities on axial T1WI image (b), equal enhancement as adjacent myometrium on post-contrast fat saturated T1WI (c), and mild heterogenous high signal intensity on diffusion-weighted images suggesting diffusion restriction (d)

showed significant differences between high-grade and low-grade ESS tumors were feather-like enhancement $(P<.001)$, necrosis $(P=.017)$, and hemorrhage $(P=.050)$. No significant differences were found in ADC values between high-grade (mean \pm standard deviation, $0.99 \pm$ $\left.0.13 \times 10^{-3} \mathrm{~mm}^{2} / \mathrm{s}\right)$ and low-grade ESS $(1.09 \pm 0.120 \times$ $\left.10^{-3} \mathrm{~mm}^{2} / \mathrm{s}, P=0.190\right)$.

\section{Diagnostic accuracy of the characteristics in \\ differentiating between high- and low-grade ESS}

We tested the diagnostic accuracy of the three outstanding imaging features: feather-like enhancement pattern, necrosis, and hemorrhage (Table 4). The feather-like enhancement yielded a diagnostic accuracy of $95 \%$ in differentiating between high-grade and low-grade ESS; this feature yielded significantly superior differentiation than necrosis $(80 \%, P=.033)$ or hemorrhage $(75 \%, P=.007)$. It had an AUC of 0.955 , which was significantly superior to that of necrosis (0.788) or hemorrhage (0.732, $P=.045)$ (Fig. 5).

\section{Discussion}

In the present study, we found that the presence of the feather-like enhancement was the most accurate MR imaging characteristic of high-grade ESS, and this feature was absent in low-grade ESS. CE MR imaging yielded significantly higher diagnostic accuracy and specificity in differentiating between high-grade and low-grade ESS compared with hemorrhage on T1-weighted or necrosis on T2-weighted MR imaging. The excellent agreement between junior and senior readers supported CE MR imaging as an objective method for preoperative MR evaluation. The feather-like enhancement likely indicated destructive myometrial invasion in high-grade ESS in contrast to the finger-like invasive projections in low-grade ESS [19]. In addition, CE MR imaging can be useful in distinguishing ESS from other uterine sarcomas. Uterine leiomyosarcomas or smooth muscle tumors with uncertain malignant potential (STUMP) exhibit pocket-like central nonenhanced areas that correlate with areas of coagulative necrosis $[4,20]$. By contrast, carcinosarcomas demonstrate 
Table 3 MR imaging characteristics between high-grade and low-grade endometrial stromal sarcomas

\begin{tabular}{|c|c|c|c|c|}
\hline Parameters & High-grade & Low-grade & $P$ value & Kappa \\
\hline$n$ & 11 & 9 & & \\
\hline Tumor size $(\mathrm{cm})$ & $11.25 \pm 2.90$ & $7.76 \pm 3.55$ & 0.056 & 0.828 \\
\hline ADC value $\left(\times 10^{-3} \mathrm{~mm}^{2} / \mathrm{sec}\right)$ & $0.99 \pm 0.13$ & $1.09 \pm 0.120$ & 0.190 & N/A \\
\hline Tumor location & & & 0.206 & 0.971 \\
\hline Fundus & $0(0.0)$ & $3(33.3)$ & & \\
\hline Body & $5(45.5)$ & $3(33.3)$ & & \\
\hline Cervix & $1(9.1)$ & $0(0.0)$ & & \\
\hline Diffuse & $5(45.5)$ & $3(33.3)$ & & \\
\hline Tumor involvement & & & 0.336 & 1.000 \\
\hline Endometrium and myometrium & $8(72.7)$ & $5(55.6)$ & & \\
\hline Endometrium only & $0(0.0)$ & $0(0.0)$ & & \\
\hline Myometrium only & $2(18.2)$ & $4(44.4)$ & & \\
\hline Cervix & $1(9.1)$ & $0(0.0)$ & & \\
\hline Serosa penetration & $5(45.5)$ & $1(11.1)$ & 0.157 & 0.870 \\
\hline Margin & & & 0.714 & 0.875 \\
\hline Well-defined & $7(63.6)$ & $5(55.6)$ & & \\
\hline IIl-defined & $4(36.4)$ & $4(44.4)$ & & \\
\hline Worm-like nodules & $4(36.4)$ & $4(44.4)$ & 1.000 & 0.898 \\
\hline Marginal nodules & $9(81.8)$ & $5(55.6)$ & 0.336 & 0.875 \\
\hline Intratumoral nodules & $10(90.9)$ & $6(66.7)$ & 0.285 & 0.828 \\
\hline T2 hypointense bands & $11(100.0)$ & $9(100.0)$ & N/A & 1.000 \\
\hline Feather-like enhancement & $10(90.9)$ & $0(0.0)$ & $<0.001 *$ & 1.000 \\
\hline Necrosis & $10(90.9)$ & $3(33.3)$ & $0.017 *$ & 0.780 \\
\hline Hemorrhage & $10(90.9)$ & $4(44.4)$ & $0.050 *$ & 0.886 \\
\hline Tumor enhancement & & & 0.082 & 0.920 \\
\hline Less than myometrium & $10(90.9)$ & $4(44.5)$ & & \\
\hline Equal to myometrium & $1(9.1)$ & $3(33.3)$ & & \\
\hline Greater than myometrium & $0(0.0)$ & $2(22.2)$ & & \\
\hline
\end{tabular}

Data in parentheses are percentages

*Statistically significant

Table 4 Diagnostic accuracy in differentiating between high-grade and low-grade endometrial stromal sarcomas based on selected MR imaging features

\begin{tabular}{lllllllllll}
\hline Parameters & $\mathrm{n}$ & $\mathrm{TP}$ & $\mathrm{TN}$ & $\mathrm{FP}$ & $\mathrm{FN}$ & Sensitivity & Specificity & Accuracy & PPV & NPV \\
\hline Feather-like enhancement & 20 & 10 & 9 & 0 & 1 & $90.9(58.7-99.8)$ & $100(100-100)$ & $95.0(75.1-99.9)$ & $100(100-100)$ & $90.0(55.5-99.7)$ \\
$\quad$ Reader 1 & 20 & 10 & 9 & 0 & 1 & $90.9(58.7-99.8)$ & $100(100-100)$ & $95.0(75.1-99.9)$ & $100(100-100)$ & $90.0(55.5-99.7)$ \\
$\quad$ Reader 2 & 20 & 10 & 9 & 0 & 1 & $90.9(58.7-99.8)$ & $100(100-100)$ & $95.0(75.1-99.9)$ & $100(100-100)$ & $90.0(55.5-99.7)$ \\
Necrosis & 20 & 10 & 6 & 3 & 1 & $90.9(58.7-99.8)$ & $66.7(29.9-92.5)$ & $80.0(56.3-94.3)$ & $76.9(46.2-95.0)$ & $85.7(42.1-99.6)$ \\
$\quad$ Reader 1 & 20 & 10 & 6 & 3 & 1 & $90.9(58.7-99.8)$ & $66.7(29.9-92.5) *$ & $80.0(56.3-94.3) *$ & $76.9(46.2-95.0)$ & $85.7(42.1-99.6)$ \\
Reader 2 & 20 & 10 & 6 & 3 & 1 & $90.9(58.7-99.8)$ & $66.7(29.9-92.5) *$ & $80.0(56.3-94.3) *$ & $76.9(46.2-95.0)$ & $85.7(42.1-99.6)$ \\
Hemorrhage & 20 & 10 & 5 & 4 & 1 & $90.9(58.7-99.8)$ & $55.6(21.2-86.3)$ & $75.0(50.9-91.3)$ & $71.4(41.9-91.6)$ & $83.3(35.9-99.6)$ \\
$\quad$ Reader 1 & 20 & 10 & 5 & 4 & 1 & $90.9(58.7-99.8)$ & $55.6(21.2-86.3) *$ & $75.0(50.9-91.3) *$ & $71.4(41.9-91.6)$ & $83.3(35.9-99.6)$ \\
Reader 2 & 20 & 9 & 5 & 4 & 2 & $81.8(48.2-97.7)$ & $55.6(21.2-86.3) *$ & $70.0(45.7-88.1) *$ & $69.2(38.6-90.9)$ & $71.4(29.0-96.3)$ \\
\hline
\end{tabular}




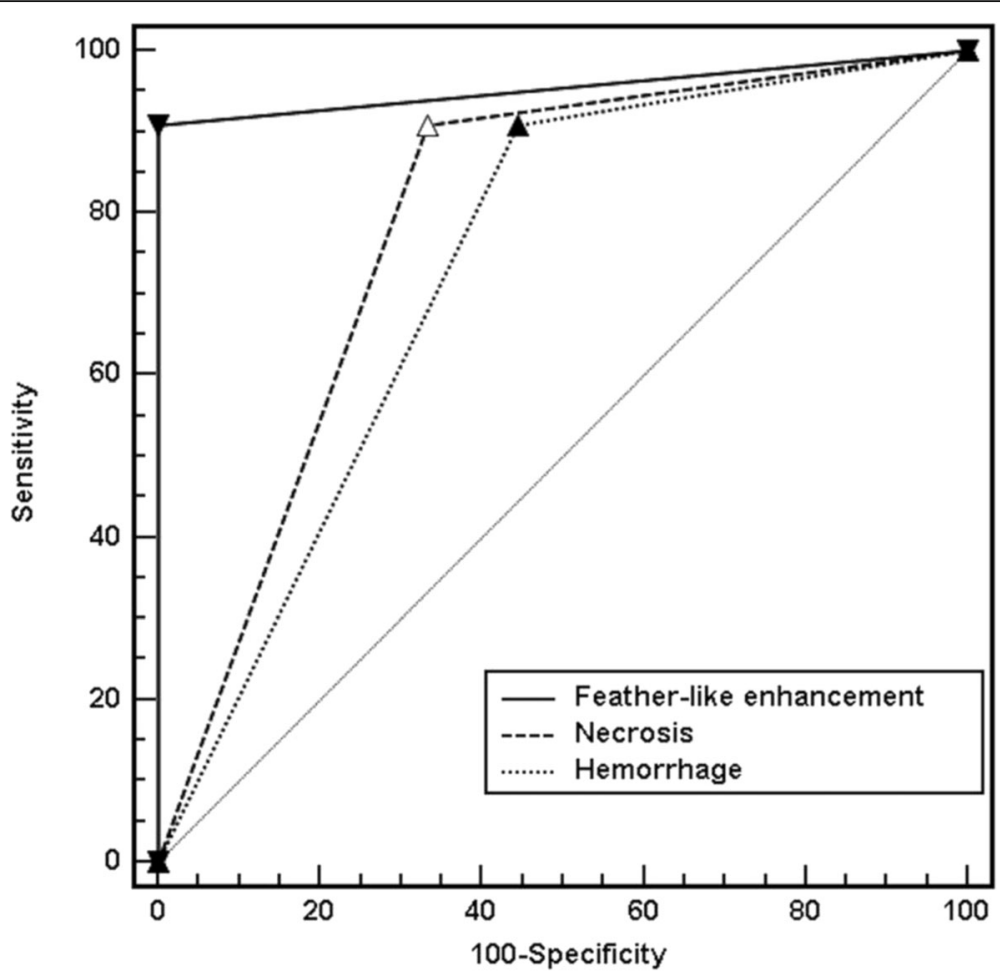

Fig. 5 Areas under the receiver operating characteristic curve (AUC) analysis to compare diagnostic performances. Feather-like enhancement demonstrated an AUC of 0.955 , significantly higher than that of necrosis $(0.788)$ or hemorrhage $(0.732, P=0.045)$, in differentiating low-grade from high-grade endometrial stromal sarcoma (ESS)

prolonged tumoral enhancement exceeding that of the myometrium [21-24]. Leiomyomas with hyaline degeneration contain numerous bands of hyalinized collagen interlacing viable cells and the necrotic focus to support the structure [25], and entire leiomyoma tumors exhibit scattered nonenhanced areas on CE MR imaging [4]. The feather-like enhancement was found in all cases in published data [13, 15, 26-28], as summarized in Table 5 . Despite the rarity of ESS, we managed to recruit the largest patient cohort to date. To the best of our knowledge, no previous study has been focusing on the pre-treatment differentiation of low-grade and high-grade ESS using MR imaging.

The T2 hypointense band is a common MR imaging feature for both high-grade and low-grade ESS; it represents preserved myometrial bundles separated by clusters of tumor cells permeating the myometrium [14]. Furukawa et al. described a T2 low-signal-intensity rim that might be the fibrous tissue layer located between viable tumor cells and the normal myometrium [29], which was absent in our imaging studies. Notably, intratumoral T2 hyperintensity is characteristic not only of ESS but also of benign degenerative leiomyomas, leiomyosarcomas or STUMP [30], and carcinosarcomas [21-23]. The T1-weighted hyperintensity observed in high-grade ESS, which represents hemorrhage [19], has also been observed in leiomyosarcomas or STUMP [20] and carcinosarcomas [23, 24]. The worm-like or marginal nodules in ESS were once considered invasive features representing intra-lymphatic or intravascular involvement [15]; however, in our study, they appeared in both high-grade and low-grade ESS and showed no statistically significant difference between the two groups. ESS could show great vessel invasion into the inferior vena cava, heart or pulmonary vessels [31-34], but only one of our cases demonstrated uterine vein invasion on MR imaging. This may be because the cohort included early stage cases.

DW imaging helps in the differentiation of malignant from benign in both pre- and post-treatment imaging studies [35]. The post-treatment follow-up using advanced diffusion-weighted imaging modules in gynecological oncologic cancers is a novel idea that has not been well studied yet. This article focuses mainly on the pre-treatment diagnosis and how the pre-treatment imaging can help to guide the clinical decision making in treatment.

Our study showed that tumor ADC values were significantly lower in ESS than in T2-hyperintense leiomyomas but were not significantly different between high-grade and low-grade ESS. Histopathologically, high-grade ESS is characterized by marked cytological 
Table 5 Literature summary of MR imaging findings of high-grade and low-grade endometrial stromal sarcomas

\begin{tabular}{|c|c|c|c|c|c|c|c|c|c|c|c|}
\hline \multirow[t]{2}{*}{ Author } & \multirow[t]{2}{*}{ Year } & \multicolumn{2}{|c|}{$\begin{array}{l}\text { Patient number } \\
\text { (Age, years) }\end{array}$} & \multirow[t]{2}{*}{$\begin{array}{l}\text { Size } \\
(\mathrm{cm})\end{array}$} & \multicolumn{2}{|c|}{ Feather-like enhancement } & \multicolumn{2}{|c|}{ Necrosis } & \multicolumn{2}{|c|}{ Hemorrhage } & \multirow[t]{2}{*}{ Reference } \\
\hline & & $\mathrm{HG}$ & LG & & $\mathrm{HG}$ & LG & $\mathrm{HG}$ & LG & $\mathrm{HG}$ & $\mathrm{LG}$ & \\
\hline Koyama & 1999 & $2(50,64)$ & $6(20-49)$ & $3-18$ & $\mathrm{~N} / \mathrm{A}$ & - & $+/-$ & - & $+/-$ & - & {$[14]$} \\
\hline La Fianza & 1999 & $1(55)$ & 0 & 11 & + & N/A & + & N/A & + & N/A & [26] \\
\hline Ueda & 2000 & $1(53)$ & $1(47)$ & $8.5,9$ & + & - & + & - & N/A & - & {$[27]$} \\
\hline Gandolfo & 2000 & $1(52)$ & $1(69)$ & 10,10 & + & N/A & + & + & + & + & [13] \\
\hline Ueda & 2001 & $4(35-55)$ & $4(53-66)$ & $1-19$ & + & - & $+/-$ & $+/-$ & $+/-$ & $+/-$ & {$[15]$} \\
\hline Toprak & 2004 & 0 & $1(24)$ & N/A & N/A & - & N/A & - & $\mathrm{N} / \mathrm{A}$ & - & [40] \\
\hline Chien & 2005 & 0 & $1(46)$ & N/A & N/A & - & N/A & - & N/A & - & {$[41]$} \\
\hline Kusaka & 2006 & 1 (39) & 0 & 8 & N/A & N/A & + & N/A & + & N/A & {$[42]$} \\
\hline Hayasaka & 2006 & $1(74)$ & 0 & N/A & + & N/A & + & N/A & + & N/A & {$[28]$} \\
\hline Tamai & 2008 & 0 & $2(40,47)$ & 5,10 & N/A & N/A & N/A & - & $\mathrm{N} / \mathrm{A}$ & - & [16] \\
\hline Fujii & 2010 & 0 & $2(44,53)$ & N/A & N/A & - & N/A & - & N/A & - & [39] \\
\hline Furukawa & 2010 & 0 & $3(20-57)$ & $5.5-11$ & N/A & - & N/A & - & N/A & $+/-$ & [29] \\
\hline Liu & 2015 & $1(73)$ & 0 & 5.2 & N/A & N/A & + & N/A & + & $\mathrm{N} / \mathrm{A}$ & [43] \\
\hline
\end{tabular}

+ = present, - = absent, N/A Not available, HG High-grade, LG Low-grade

atypia, nuclear pleomorphism, high mitotic activity, and extensive invasion of sarcomatous components [36], whereas low-grade ESS is characterized by densely uniform stromal cells with minimal cellular pleomorphism and mild nuclear atypia [5]. The ADC values in ESS are influenced by the nuclear-to-cytoplasm ratio and cellular density in both stromal and sarcoma components [16, 37]. Hence, the ADC values of ESS are not indicative of the aggressiveness of ESS. The results of our study are in line with those of other studies on uterine sarcomas such as leiomyosarcomas or STUMP [4], carcinosarcomas [24, 38], and ESS [16, 39], which also showed lower ADC values for ESS than for the myometrium.

This study had limitations. First, this was a retrospective study. In addition, given the long 17-year study span, the MR scanners and parameters varied. Second, during the study period, three ESS classification guidelines were applied, and we adapted the most recent 2014 WHO classification [18]. The actual frequency and clinical features of the new category, that is high-grade ESS, are unknown. Future studies should apply the new WHO classification system to determine clinical, imaging, pathological, and molecular differences between lowgrade ESS, high-grade ESS, and undifferentiated uterine sarcomas to understand the prognostic significance of MR imaging.

\section{Conclusions}

DW MR imaging is useful in diagnosing ESS against T2hyperintense leiomyomas, whereas contrast enhancement aids in further differentiating between high- and low-grade
ESS. Pretreatment differentiation between high-grade and low-grade ESS based on MR imaging would assist clinicians in selecting the most appropriate treatment plan.

\section{Additional file}

Additional file 1: Table S1. List of MR imaging features analyzed in the present study. (DOCX $18 \mathrm{~kb})$

\section{Abbreviations}

ADC: Apparent diffusion coefficient; AUC: Areas under the receiver operating characteristic curve; CE: Contrast-enhanced; DW: Diffusion-weighted; ESS: Endometrial stromal sarcoma; MR: Magnetic resonance; PACS: Picture archiving and communication system; STUMP: Smooth muscle tumors with uncertain malignant potential; WHO: World Health Organization

\section{Acknowledgements}

The authors acknowledge the assistance provided by the Cancer Center and Clinical Trial Center (Statistician Dr. Lan-Yan Yang), Chang Gung Memorial Hospital, Linkou, Taiwan, which was founded by the Ministry of Health and Welfare of Taiwan MOHW106-TDU-B-212-113005.

\section{Authors' contributions}

YLH: data acquisition, literature research, and manuscript preparation. SHU: pathology review. KC, YTH and KKN: data acquisition and review. HYL: data analysis. TCC and CHL: study conception and design and manuscript editing. GL: study conception and design, manuscript review and guarantor of integrity of the entire study. All authors approved the final manuscript.

\section{Funding}

This study was funded by the National Science Council, Taiwan (grant no.: NSC 103-2314-B-182A-006), Ministry of Science and Technology, Taiwan (grant no.: MOST 104-2314-B-182A-095-MY3) and Chang Gung Medical Foundation grant CIRPG3E0022, CPRPG3G0023, CIRPG3H0012, Chang Gung IRB 104-8300, 201701215B0.

Availability of data and materials Not applicable. 


\section{Ethics approval and consent to participate}

This was a HIPAA-compliant retrospective study with the institutional review board-approval. The requirement for informed consent was waived due to the retrospective nature of the study.

\section{Consent for publication}

Not applicable.

\section{Competing interests}

The authors declare that they have no competing interests.

\section{Author details}

${ }^{1}$ Department of Medical Imaging and Intervention, Chang Gung Memorial Hospital at Linkou, 5 Fuhsing St., Guishan, Taoyuan, Taiwan33382. ${ }^{2}$ Imaging Core Laboratory, Institute for Radiological Research, Chang Gung Memorial Hospital at Linkou and Chang Gung University, 5 Fuhsing St., Guishan, Taoyuan, Taiwan33382. ${ }^{3}$ Department of Pathology, Chang Gung Memorial Hospital at Linkou and Chang Gung University, 5 Fuhsing St., Guishan, Taoyuan, Taiwan33382. ${ }^{4}$ Department of Obstetrics and Gynecology and Gynecologic Cancer Research Center, Chang Gung Memorial Hospital at Linkou and Chang Gung University, 5 Fuhsing St., Guishan, Taoyuan, Taiwan33382. ${ }^{5}$ Department of Diagnostic Radiology, Chang Gung Memorial Hospital at Keelung, 222, Maijin Rd, Keelung, Taiwan20401. ${ }^{6}$ Clinical Metabolomics Core Laboratory, Chang Gung Memorial Hospital at Linkou, 5 Fuhsing St., Guishan, Taoyuan, Taiwan33382. ${ }^{7}$ Clinical Trial Center, Chang Gung Memorial Hospital at Linkou and Chang Gung University, 5 Fuhsing St., Guishan, Taoyuan, Taiwan33382.

\section{Received: 24 March 2019 Accepted: 13 August 2019} Published online: 12 September 2019

\section{References}

1. National Comprehensive Cancer Network. NCCN Clinical Practice Guidelines in Oncology: Uterine Neoplasms (Version 3.2019). http://www.ncen.org/ professionals/physician_gls/pdf/uterine.pdf. Accessed 18 Mar 2019.

2. D'Angelo E, Prat J. Uterine sarcomas: a review. Gynecol Oncol. 2010;116: 131-9.

3. Nordal RR, Thoresen SO. Uterine sarcomas in Norway 1956-1992: incidence, survival and mortality. Eur J Cancer. 1997;33:907-11.

4. Lin G, Yang LY, Huang YT, Ng KK, Ng SH, Ueng SH, et al. Comparison of the diagnostic accuracy of contrast-enhanced MRI and diffusion-weighted MRI in the differentiation between uterine leiomyosarcoma / smooth muscle tumor with uncertain malignant potential and benign leiomyoma. J Magn Reson Imaging. 2016:43:333-42.

5. Tavassoli FA, Devilee P. World Health Organization Classification of Tumours Pathology and Genetics of Tumours of the Breast and Female Genital Organs. Lyon: IARC Press; 2003. p. 233-6.

6. Kurihara S, Oda Y, Ohishi Y, Iwasa A, Takahira T, Kaneki E, et al. Endometrial stromal sarcomas and related high-grade sarcomas: immunohistochemical and molecular genetic study of 31 cases. Am J Surg Pathol. 2008;32:1228-38.

7. Seagle BL, Shilpi A, Buchanan S, Goodman C, Shahabi S. Low-grade and high-grade endometrial stromal sarcoma: a National Cancer Database study. Gynecol Oncol. 2017;146:254-62.

8. Gadducci A, Cosio S, Romanini A, Genazzani AR. The management of patients with uterine sarcoma: a debated clinical challenge. Crit Rev Oncol Hematol. 2008;65:129-42.

9. Sala E, Rockall AG, Freeman SJ, Mitchell DG, Reinhold C. The added role of MR imaging in treatment stratification of patients with gynecologic malignancies: what the radiologist needs to know. Radiology. 2013;266:717-40.

10. Razek AAKA, El-Serougy L, Abdelsalam M, Gaballa G, Talaat M. Differentiation of residual/recurrent gliomas from postradiation necrosis with arterial spin labeling and diffusion tensor magnetic resonance imaging-derived metrics. Neuroradiology. 2018;60:169-77.

11. Abdel Razek AAK, Zaky M, Bayoumi D, Taman S, Abdelwahab K, Alghandour R. Diffusion tensor imaging parameters in differentiation recurrent breast cancer from post-operative changes in patients with breast-conserving surgery. Eur J Radiol. 2019;111:76-80.
12. Ueda H, Togashi K, Konishi I, Kataoka ML, Koyama T, Fujiwara T, et al. Unusual appearances of uterine leiomyomas: MR imaging findings and their histopathologic backgrounds. Radiographics. 1999;19:S131-45.

13. Gandolfo N, Gandolfo NG, Serafini G, Martinoli C. Endometrial stromal sarcoma of the uterus: MR and US findings. Eur Radiol. 2000;10:776-9.

14. Koyama T, Togashi K, Konishi I, Kobayashi H, Ueda H, Kataoka ML, et al. MR imaging of endometrial stromal sarcoma: correlation with pathologic findings. AJR Am J Roentgenol. 1999;173:767-72.

15. Ueda M, Otsuka M, Hatakenaka M, Sakai S, Ono M, Yoshimitsu K, et al. MR imaging findings of uterine endometrial stromal sarcoma: differentiation from endometrial carcinoma. Eur Radiol. 2001;11:28-33.

16. Tamai K, Koyama T, Saga T, Morisawa N, Fujimoto K, Mikami Y, et al. The utility of diffusion-weighted MR imaging for differentiating uterine sarcomas from benign leiomyomas. Eur Radiol. 2008;18:723-30.

17. Tirumani SH, Ojili V, Shanbhogue AK, Fasih N, Ryan JG, Reinhold C. Current concepts in the imaging of uterine sarcoma. Abdom Imaging. 2013:38:397-411.

18. Kurman RJ, Carcangiu ML, Herrington CS, Young RH, editors. WHO classification of Tumours of female reproductive organs. 4th ed. Lyon: IARC Press; 2014. p. 141-5.

19. Amant F, Coosemans $A$, Debiec-Rychter M, Timmerman D, Vergote I. Clinical management of uterine sarcomas. Lancet Oncol. 2009;10:1188-98.

20. Tanaka YO, Nishida M, Tsunoda H, Okamoto Y, Yoshikawa H. Smooth muscle tumors of uncertain malignant potential and leiomyosarcomas of the uterus: MR findings. J Magn Reson Imaging. 2004;20:998-1007.

21. Bharwani N, Newland A, Tunariu N, Babar S, Sahdev A, Rockall AG, et al. MRI appearances of uterine malignant mixed mullerian tumors. AJR Am J Roentgenol. 2010;195:1268-75.

22. Hernandez Mateo P, Mendez Fernandez R, Serrano TE. Uterine sarcoma vs adenocarcinoma: can MRI distinguish between them? Radiologia. 2016:58:199-206.

23. Tanaka YO, Tsunoda H, Minami R, Yoshikawa H, Minami M. Carcinosarcoma of the uterus: MR findings. J Magn Reson Imaging. 2008;28:434-9.

24. Huang YT, Chang CB, Yeh CJ, Lin G, Huang HJ, Wang CC, et al. Diagnostic accuracy of 3.0T diffusion-weighted MRI for patients with uterine carcinosarcoma: Assessment of tumor extent and lymphatic metastasis. J Magn Reson Imaging. 2018;48:622-31.

25. Bell SW, Kempson RL, Hendrickson MR. Problematic uterine smooth muscle neoplasms. A clinicopathologic study of 213 cases. Am J Surg Pathol. 1994;18:535-58.

26. La Fianza A, Meloni G, Alberici E, Campani R. Magnetic resonance appearance of endometrial sarcoma: report of a case with unusual findings. Magn Reson Imaging. 1999;17:637-40.

27. Ueda M, Otsuka M, Hatakenaka M, Torii Y. Uterine endometrial stromal sarcoma located in uterine myometrium: MRI appearance. Eur Radiol. 2000;10:780-2.

28. Hayasaka K, Morita K, Saitoh T, Tanaka Y. Uterine adenofibroma and endometrial stromal sarcoma associated with tamoxifen therapy: MR findings. Comput Med Imaging Graph. 2006;30:315-8.

29. Furukawa R, Akahane M, Yamada H, Kiryu S, Sato J, Komatsu S, et al. Endometrial stromal sarcoma located in the myometrium with a lowintensity rim on T2-weighted images: report of three cases and literature review. J Magn Reson Imaging. 2010;31:975-9.

30. Yamashita Y, Torashima M, Takahashi M, Tanaka N, Katabuchi H, Miyazaki K, et al. Hyperintense uterine leiomyoma at T2-weighted MR imaging: differentiation with dynamic enhanced MR imaging and clinical implications. Radiology. 1993;189:721-5.

31. Boskovic V, Bozanovic T, Ljubic A, Likic-Ladjevic I, Janjic T, Milicevic S. Endometrial stromal sarcoma with intracaval extension at initial presentation. Eur J Gynaecol Oncol. 2013;34:280-1.

32. Gabal S, Ashour Z, Hamada G, Aziz SA, Khairy H, Badawy H, et al. Low-grade endometrial stromal sarcoma with intravenous extension to the heart. Medscape J Med. 2009;11:23.

33. Yokoyama Y, Ono Y, Sakamoto T, Fukuda I, Mizunuma H. Asymptomatic intracardiac metastasis from a low-grade endometrial stromal sarcoma with successful surgical resection. Gynecol Oncol. 2004;92:999-1001.

34. Veroux P, Veroux M, Nicosia A, Bonanno MG, Tumminelli MG, Milone P, et al. Thrombectomy of the inferior vena cava from recurrent low-grade endometrial stromal sarcoma: case report and review of the literature. J Surg Oncol. 2000;74:45-8. 
35. Abdel Razek AAK. Routine and Advanced Diffusion Imaging Modules of the Salivary Glands. Neuroimaging Clin N Am. 2018;28:245-54.

36. Lee $\mathrm{CH}$, Nucci MR. Endometrial stromal sarcoma--the new genetic paradigm. Histopathology. 2015;67:1-19.

37. Lin YC, Lin G, Hong JH, Lin YP, Chen FH, Ng SH, et al. Diffusion radiomics analysis of intratumoral heterogeneity in a murine prostate cancer model following radiotherapy: Pixelwise correlation with histology. J Magn Reson Imaging. 2017:46:483-9.

38. Takeuchi M, Matsuzaki K, Harada M. Carcinosarcoma of the uterus: MRI findings including diffusion-weighted imaging and MR spectroscopy. Acta Radiol. 2016:57:1277-84.

39. Fujii S, Kaneda S, Tsukamoto K, Kakite S, Kanasaki Y, Matsusue E, et al. Diffusion-weighted imaging of uterine endometrial stromal sarcoma: a report of 2 cases. J Comput Assist Tomogr. 2010;34:377-9.

40. Toprak U, Pasaoglu E, Karademir MA, Gulbay M. Sonographic, CT, and MRI findings of endometrial stromal sarcoma located in the myometrium and associated with peritoneal inclusion cyst. AJR Am J Roentgenol. 2004;182:1531-3.

41. Chien JC, Hsieh SC, Lee RC, Chen CY, Cheng CJ, Chan WP. Endometrial stromal sarcoma mimicking submucosal myoma protruding to the vagina: MRI findings. Eur J Gynaecol Oncol. 2005;26:657-60.

42. Kusaka M, Mikuni M, Nishiya M. A case of high-grade endometrial stromal sarcoma arising from endometriosis in the cul-de-sac. Int J Gynecol Cancer. 2006;16:895-9.

43. Liu G, Zhang C, Ma Z, Zhang Q, Liu B. Endometrial stromal sarcoma with endometrioid adenocarcinoma of the uterus: a case report. Int J Clin Exp Pathol. 2015;8:5242-446.

\section{Publisher's Note}

Springer Nature remains neutral with regard to jurisdictional claims in published maps and institutional affiliations.

Ready to submit your research? Choose BMC and benefit from:

- fast, convenient online submission

- thorough peer review by experienced researchers in your field

- rapid publication on acceptance

- support for research data, including large and complex data types

- gold Open Access which fosters wider collaboration and increased citations

- maximum visibility for your research: over $100 \mathrm{M}$ website views per year

At BMC, research is always in progress.

Learn more biomedcentral.com/submissions 